\title{
Experience with using counterexamples in an introductory calculus
}

class

Sergiy Klymchuk

School of Computer and Mathematical Sciences, Auckland University of Technology, Auckland, New Zealand

Corresponding author:

Dr Sergiy Klymchuk

Associate Professor

School of Computer and Mathematical Sciences

Faculty of Design and Creative Technologies

Auckland University of Technology

Private Bag 92006

Auckland 1142

New Zealand

Tel: +64-9-921 9999 ext.8431

Fax: +64-9-921 9944

E-mail: sergiy.klymchuk@aut.ac.nz 


\section{Experience with using counterexamples in an introductory calculus course}

The paper describes a personal experience with using counterexamples as a pedagogical strategy in the teaching and learning of an introductory calculus course at a university of technology.

Keywords: calculus; counterexamples; teaching; learning

Deciding on an assertion's validity is important in the information age. A counterexample can quickly and easily show that a given statement is false. Counterexamples thus offer powerful and effective tools for mathematicians, scientists, and researchers. They can indicate that a hypothesis is wrong. Before attempting to find proof for an assertion, looking for counterexamples may save an investigator lots of time and effort. Examples from history of mathematics in a class can be both informative and entertaining for the students. Below are three famous examples that I normally use in my classes.

Example 1. For a long time mathematicians tried to find a formula which generated only prime numbers. Numbers of the form $2^{2^{n}}+1$, where $n$ is a natural number were once believed to all be prime, until Euler found a counterexample. He showed that for $n=5$ that number is composite: $2^{2^{5}}+1=641 \times 6700417$.

Example 2. Another conjecture about prime numbers is still awaiting proof or disproof. The Goldbach-Euler conjecture, posed by Goldbach in a 1742 letter to Euler, looks deceptively simple: every even integer greater than 2 is the sum of two prime numbers. For example, $12=5+7,20=3+17$, and so on. Powerful computers were used to search for possible counterexamples and they found none among $4,6, \ldots, 4 \times 10^{14}[1]$. In 2000 the book publishing company Faber \& Faber 
offered a US\$1 million prize to anyone who could prove or disprove this conjecture within two years. The prize went unclaimed.

Example 3. In the $19^{\text {th }}$ century the great German mathematician Weierstrass constructed his famous counterexamples to this statement: If a function is continuous on $(a, b)$, then it is differentiable at some points on $(a, b)$. Many mathematicians at that time thought that such 'monster-functions' that were continuous but not differentiable at any point were absolutely useless for practical applications. About a hundred years later Norbert Wiener, the founder of cybernetics pointed out in [2] that such curves exist in nature - for example, they are trajectories of particles in Brownian motion. In recent decades such curves have been investigated in the theory of fractals - a fast growing area with many applications.

Dealing with counterexamples for the first time can be challenging for students. When they hear they can disprove a wrong statement by providing one counterexample, many students think they can "prove” a correct statement by showing an example. Even if they know they cannot prove a theorem by providing only examples, it is hard for some students to accept the fact that a single counterexample disproves a statement. Some students believe that a particular counterexample is just an exception to the rule at hand, and that no other 'pathological' cases exist. Selden \& Selden have articulated these ideas in [3]: “Students quite often fail to see a single counterexample as disproving a conjecture. This can happen when a counterexample is perceived as 'the only one that exists', rather than being seen as generic."

With experience students understand the role of counterexamples and become interested in creating them. Using counterexamples to disprove wrong statements can generate many questions for discussion. What changes will make the statement at 
hand correct? How can you change a counterexample and have it remain one? Can you think of other statements that your counterexample refutes? Can you find another type of function altogether that will be a counterexample or construct a general class of counterexamples to the statement at hand?

In developing counterexamples students are forced to pay attention to every detail in a statement - the word order, the symbols used, the shape of brackets defining intervals, whether the statement applies to a point or to an interval, and so on. Consider the following theorem.

Theorem 1. If a function $f(x)$ is differentiable on $(a, b)$ and its derivative is positive for all $x$ in $(a, b)$, then the function is increasing on $(a, b)$.

The following two statements look quite similar to Theorem 1, but both are incorrect:

Statement 1. If a function $f(x)$ is differentiable on $(a, b)$ and its derivative is positive at a point $x=c$ in $(a, b)$, then there is a neighbourhood of the point $x=c$ where the function is increasing.

Statement 2. If a function $f(x)$ is differentiable on its domain and its derivative is positive for all $x$ from its domain, then the function is increasing everywhere on its domain.

Students who find counterexamples to the last two statements must grapple with their subtle differences.

An example from my teaching practice of using counterexamples below shows how they can foster discussion. Consider the following incorrect statement. Statement 3. If a function $y=f(x)$ is defined on $[a, b]$ and continuous on $(a, b)$, then for any $N$ between $f(a)$ and $f(b)$ there is some point $c \in(a, b)$ such that $f(c)=N$. 
The only difference a student can see between Statement 3 and the Intermediate Value Theorem is in the shape of the brackets of the interval where the function is continuous: the function is continuous on an open interval $(a, b)$, instead of a closed interval $[a, b]$. When students are asked to disprove the statement they usually come up with something like this:

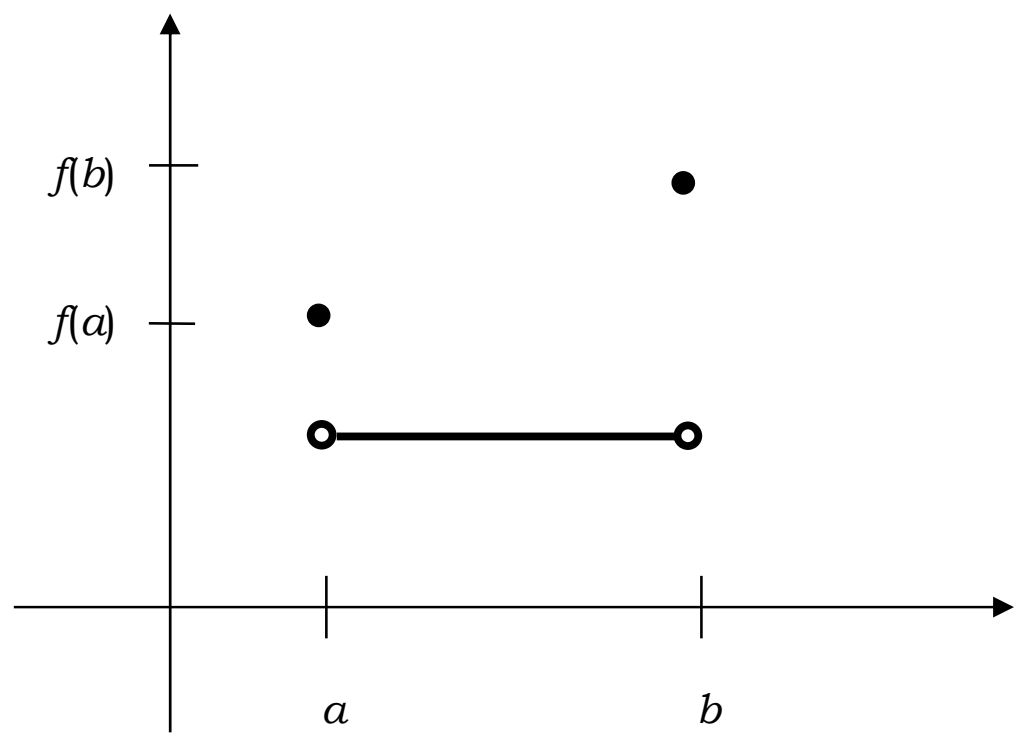

Figure 1. A possible student's counterexample to Statement 3.

To generate discussion and create other counterexamples one can suggest the students the following exercise:

Exercise 1. On the graph shown in Figure 1 the statement's conclusion is not true for any value of $N$ between $f(a)$ and $f(b)$. Modify the graph in such a way that the statement's conclusion is true for:

a) exactly one value of $N$ between $f(a)$ and $f(b)$;

b) infinitely many but not all values of $N$ between $f(a)$ and $f(b)$.

One can then expect students to sketch graphs like these: 
a)

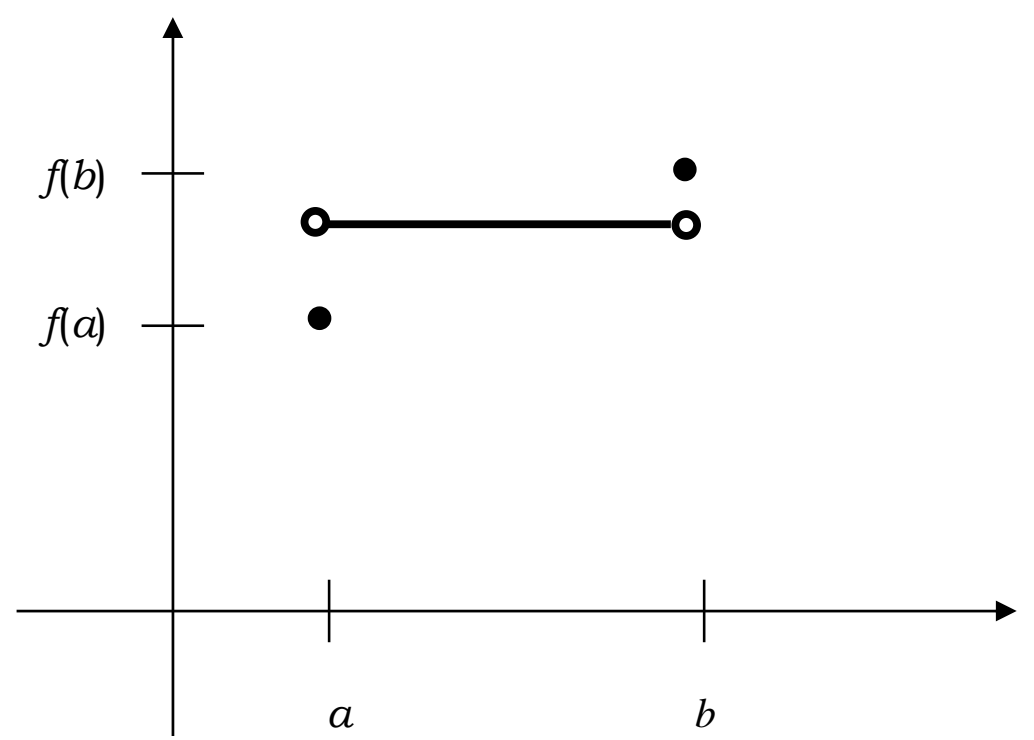

Figure 2. A possible student's answer to Exercise 1(a).

b)

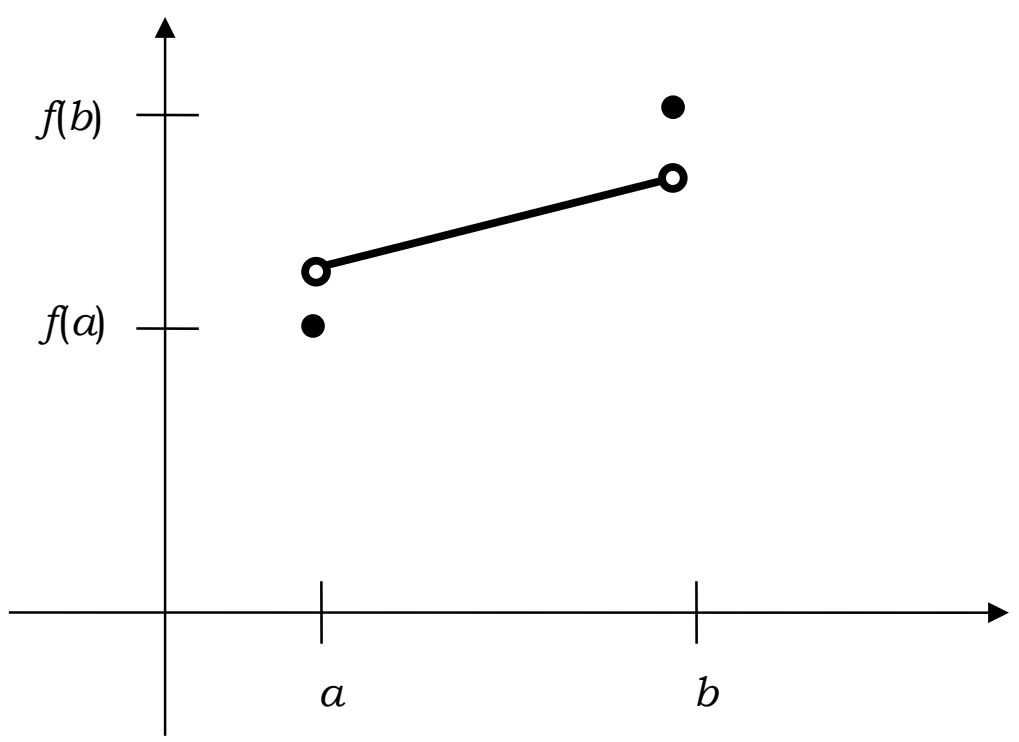

Figure 3. A possible student's answer to Exercise 1(b).

Another exercise can be suggested to the students:

Exercise 2. Give as a counterexample a graph that doesn't have 'white circles'.

The students may come up with something like this: 


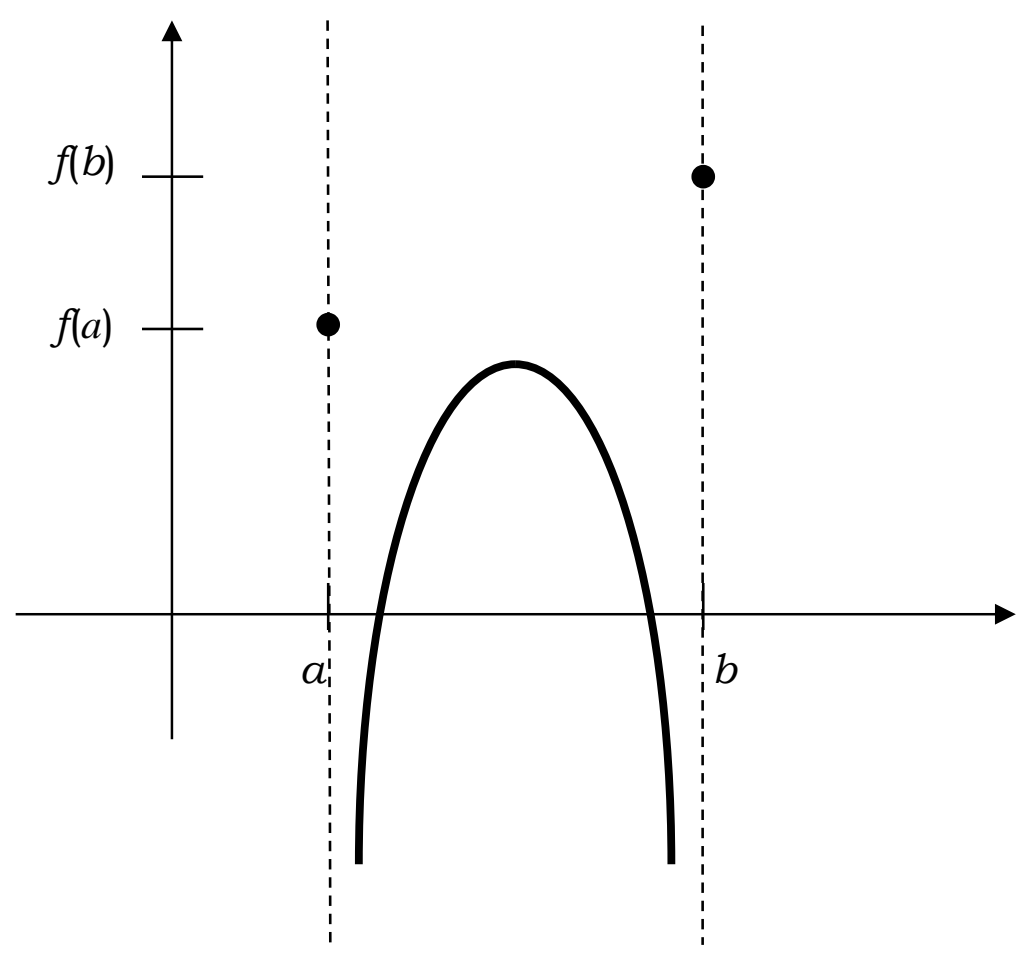

Figure 4. A possible student's answer to Exercise 2.

Below are some more examples of incorrect statements that I discuss with the students:

Statement 4. The tangent to a curve at a point is the line which touches the curve at that point but does not cross it there.

Statement 5. A function $y=f(x)$ is bounded on R if for any $x \in R$ there is $M>0$ such that $|f(x)| \leq M$.

Statement 6. If $f(x)<g(x)$ for all $x>0$ and both $\lim _{x \rightarrow \infty} f(x)$ and $\lim _{x \rightarrow \infty} g(x)$ exist then $\lim _{x \rightarrow \infty} f(x)<\lim _{x \rightarrow \infty} g(x)$

Statement 7. Every continuous and bounded function on $(-\infty, \infty)$ takes on its extreme values. 
Statement 8. If a function is continuous on the interval $(a, b)$ and its graph is a smooth curve (no sharp corners) on that interval then the function is differentiable at every point on $(a, b)$.

Statement 9. If a function $y=f(x)$ is differentiable at the point $x=a$ then its derivative is continuous at $x=a$.

Statement 10. A plane figure of an infinite area rotated about an axis always produces a solid of revolution of infinite volume.

With experience students learn that in creating counterexamples it is useful to have at their disposal a large assortment of graphs and functions with interesting properties building their 'example space' [4]. Understanding the anomalies and distinguishing features of these functions will provide them a natural starting point for developing their own counterexamples. To construct counterexamples most of the students try to use familiar graphs of polynomial functions, basic trigonometric functions and their inverses, piecewise functions (like step functions), and graphs with sharp corners (like that of the absolute value function $|x|)$ or cusps $\left(\sqrt[3]{x^{2}}\right)$. Search for possible counterexamples to harder examples can add to the student's arsenal graphs of more exotic functions like oscillations with and without damping factors (like $\sin \frac{1}{x}, x \sin \frac{1}{x}$, and $x^{2}\left|\cos \frac{\pi}{x}\right|$ ), and even more exotic functions like Dirichlet and Weierstrass functions, which are notable in the history of mathematics.

I mention here a few of the other ways I have used counterexamples in teaching. On different occasions, I have given students mixtures of correct and incorrect statements, asked students to create their own wrong statements and associated counterexamples, made deliberate errors in my lecture and moved on with the hope that students would detect them, asked students to spot errors in their 
textbook, given students extra credit for providing counterexamples to challenging statements I posed in class, and included on assignments and tests questions that require students to construct counterexamples.

Teaching experience has shown that using counterexamples fosters discovery and makes learning more active. In an international study involving more than 600 students from 10 universities in different countries [5] 92\% of the participating students found the use of counterexamples to be very effective. The students reported it helped them to understand concepts better, prevent mistakes, develop logical and critical thinking, and made learning mathematics more challenging, interesting and creative. Another (case) study [6] showed that the use of counterexamples in teaching could improve students' performance on test questions that required conceptual understanding. Apart from that study there was another indication from the same university that the usage of counterexamples was beneficial to the students. On a routine calculus course evaluation by the students in the end of the course the following statement was added to the form (the statement was formulated in the same style as other statements in the evaluation form): The usage of counterexamples in class helped me to learn. There were 54 students on that day in class who filled the evaluation form and 52 of them (96\%) ticked either 'Agree' or 'Strongly Agree' box for the above statement.

From my experience, working with counterexamples reduces some common misconceptions that can arise in calculus courses that avoid special cases and expose students only to 'nice' functions and 'good' simple examples, misconceptions explained by Tall’s generic extension principle: “If an individual works in a restricted context in which all the examples considered have a certain property, then, in the 
absence of counterexamples, the mind assumes the known properties to be implicit in other contexts.” [7].

A collection of 80+ incorrect statements from a first-year introductory calculus course and suggested counterexamples to them illustrated by graphs can be found in [8]. The book by Gelbaum \& Olmsted [9] is a classical resource for using counterexamples in advanced calculus and mathematical analysis courses.

\section{References}

[1] J. Richstein, Verifying the Goldbach conjecture up to 4·10^14, Mathematics of Computation. 70 (236) (2000), pp. 1745-1749.

[2] N. Wiener, I am a Mathematician. London, Gollancz, 1956.

[3] A. Selden and J. Selden, The role of examples in learning mathematics, Research Sampler. 5 (1998), 4 p. Available at http://www.maa.org/t and_l/sampler/rs_5.html

[4] J. Mason and A. Watson, Getting students to create boundary examples, MSOR Connections 1(1) (2001), pp. 9-11.

[5] N. Gruenwald and S. Klymchuk, Using counter-examples in teaching Calculus. The New Zealand Mathematics Magazine. 40(2) (2003), pp. 33-41.

[6] S. Klymchuk, Counter-examples in teaching/learning of Calculus: Students' performance. The New Zealand Mathematics Magazine. 42(1) (2005), pp. 31-38.

[7] D.O. Tall, The psychology of advanced mathematical thinking, in Advanced Mathematical Thinking, D.O. Tall, ed., Kluwer: Dordrecht, 1991, pp. 3-21.

[8] S. Klymchuk, Counterexamples in Calculus, Mathematical Association of America, Washington, DC, 2010.

[9] B.R. Gelbaum and J.M.H. Olmsted, Counterexamples in Analysis. Holden-Day, Inc., San Francisco, 1964. 